\title{
Embedding randomised controlled trials in renal registries
}

\author{
Fergus J Caskey', Thabiet Jardine², M Razeen Davids² \\ 'Population Health Sciences, University of Bristol and North Bristol NHS Trust, UK; '2Division of Nephrology, Stellenbosch University and \\ Tygerberg Hospital, Cape Town, South Africa.
}

\section{ABSTRACT}

Traditional randomised controlled trials that rely on research staff to collect data are becoming increasingly expensive. As a result, the number of interventions that can be scrutinised for effectiveness will be limited. Further, while results from such trials have high internal validity, they will have limited external validity - generalisability to the real-world population. One solution is to adopt a more pragmatic approach and embed randomisation into routine healthcare databases such as registries. There are a number of ways that this can be done. Most commonly, registries simply provide extended follow-up to traditional explanatory trials, but with the necessary permissions more novel approaches are possible. Registries can be used to identify potentially eligible participants, provide the baseline data and provide all of the follow-up data. Proportionate to the risk associated with the intervention, routine healthcare databases can also provide some of the safety monitoring data, greatly reducing the burden and cost of the trial. To illustrate the opportunities and challenges, a number of reported and ongoing registry trials are presented.

Keywords: registry trials; pragmatic trials; randomised controlled trials; nephrology trials.

\section{INTRODUCTION}

For decades, randomised controlled trials (RCTs) have been considered the most robust form of primary research on which to base treatment recommendations [I]. However, people with kidney disease are often excluded from trials of common conditions such as cardiovascular disease and osteoporosis [2,3]. For trials focused on kidney disease, nephrology sits at the bottom of the league table [4], and even in those trials, participants tend to be unrepresentative of the wider kidney patient population [5].

The generalisability criticism is, of course, not unique to nephrology and as far back as the 1960s it was recognised that there needed to be two different types of trials explanatory and pragmatic [6].

- Explanatory trials, which maximise the intervention's chance of demonstrating an effect through the expected mechanism, with little attention paid to the issue of whether this outcome would be achieved under real-world conditions, either locally or in more distant settings.

- Pragmatic trials, which maximise applicability of the intervention to usual care across a range of local and distant settings [7].

Another problem with RCTs is the total cost, which can run into tens to hundreds of millions of dollars on the rare occasions that it is reported [8]. Such costs limit trials to evaluations of products - pharmaceutical or device - with the potential to generate profit that will recoup those costs. Limiting RCTs to these novel interventions misses a huge opportunity to critically evaluate and evolve existing practice to optimise outcomes for patients and the healthcare economy.

One way to mitigate both the generalisability and the financial risks of RCTs in nephrology is to rely more on

Received 23 October 2019; accepted 0I November 2019; published 07 November 2019. 
routinely collected data for everything from the identification of potential participants to assessing trial outcomes. If permissions allow, these routine data could include electronic health records, prescribing databases, billing databases, device registries or disease registries. Such RCTs are hereafter referred to as 'registry trials'. This paper will first consider some of the strengths and limitations of registry RCTs before looking at examples of renal and non-renal registry RCTs that have been completed or are in progress.

\section{ADVANTAGES AND CHALLENGES OF REGISTRY RCTS}

The advantages of registry RCTs can be summarised as lower cost, enhanced generalisability, consecutive enrolment and completeness of follow-up. Figure I compares and contrasts registry-based RCTs with traditional RCTs $[9-||]$.

The costs of a registry RCT are minimised in several ways. Recruitment is often easier and quicker as the eligibility criteria are wide and many more patients can be invited to participate. Depending on information governance permissions, it may even be possible to use the registry to identify eligible patients and approach them to explore their willingness to take part. Another major saving comes from reducing traditional data capture activity by replacing the formal data collection procedures at baseline and/or follow-up with data collected routinely from one or multiple routine data sources. Depending on the intervention being tested, it may also be possible to rely on prospective routine data collection for some of the monitoring of adverse events that occur with participants like hospital admissions, deaths, serious adverse reactions (events that are considered to have been a direct effect of the intervention) and serious adverse events (events that are considered not to have been a direct effect of the intervention). The extent to which this can be done must be proportionate to what is known already about the risks associated with the intervention being tested [12]. For example, vitamin $D$ has been judged safe for the public to purchase without a prescription, so while safety monitoring is clearly required it would seem disproportionate to require expedited detailed capture of all adverse events in a trial of vitamin $\mathrm{D}$ versus placebo in dialysis patients.

A major criticism of explanatory trials is that while they may have very high internal validity - that is, in a highly selected trial cohort with limited possible external factors the intervention is effective - they have low external validity, meaning that in the patients coming to clinic today the intervention is much less likely to be effective. By having very wide eligibility criteria, registry RCTs include cohorts of patients much more representative of the real world.

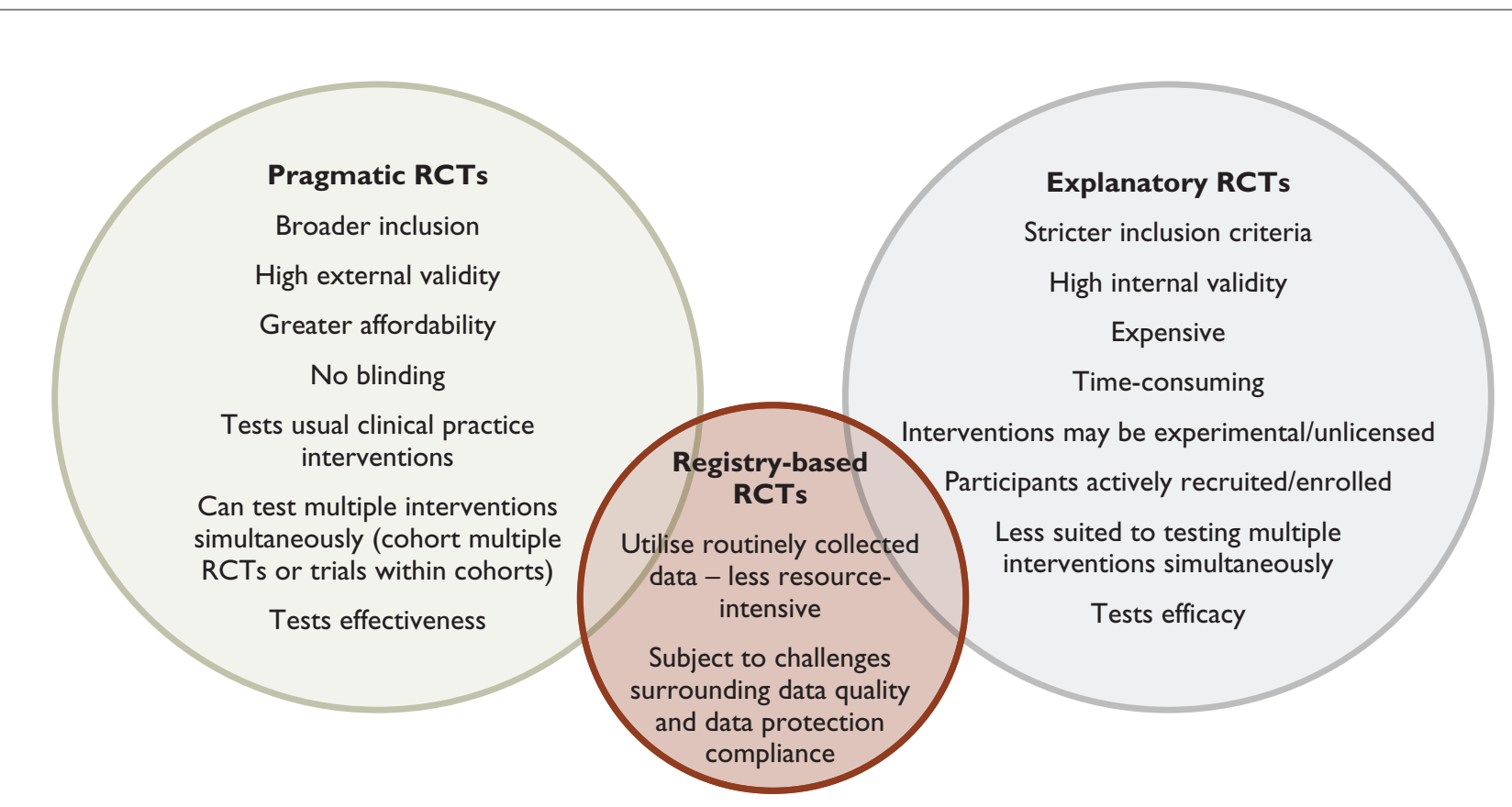

Figure I. Venn diagram illustrating the relationship between, and characteristics of, traditional pragmatic RCTs, explanatory RCTs and registry-based RCTs. 


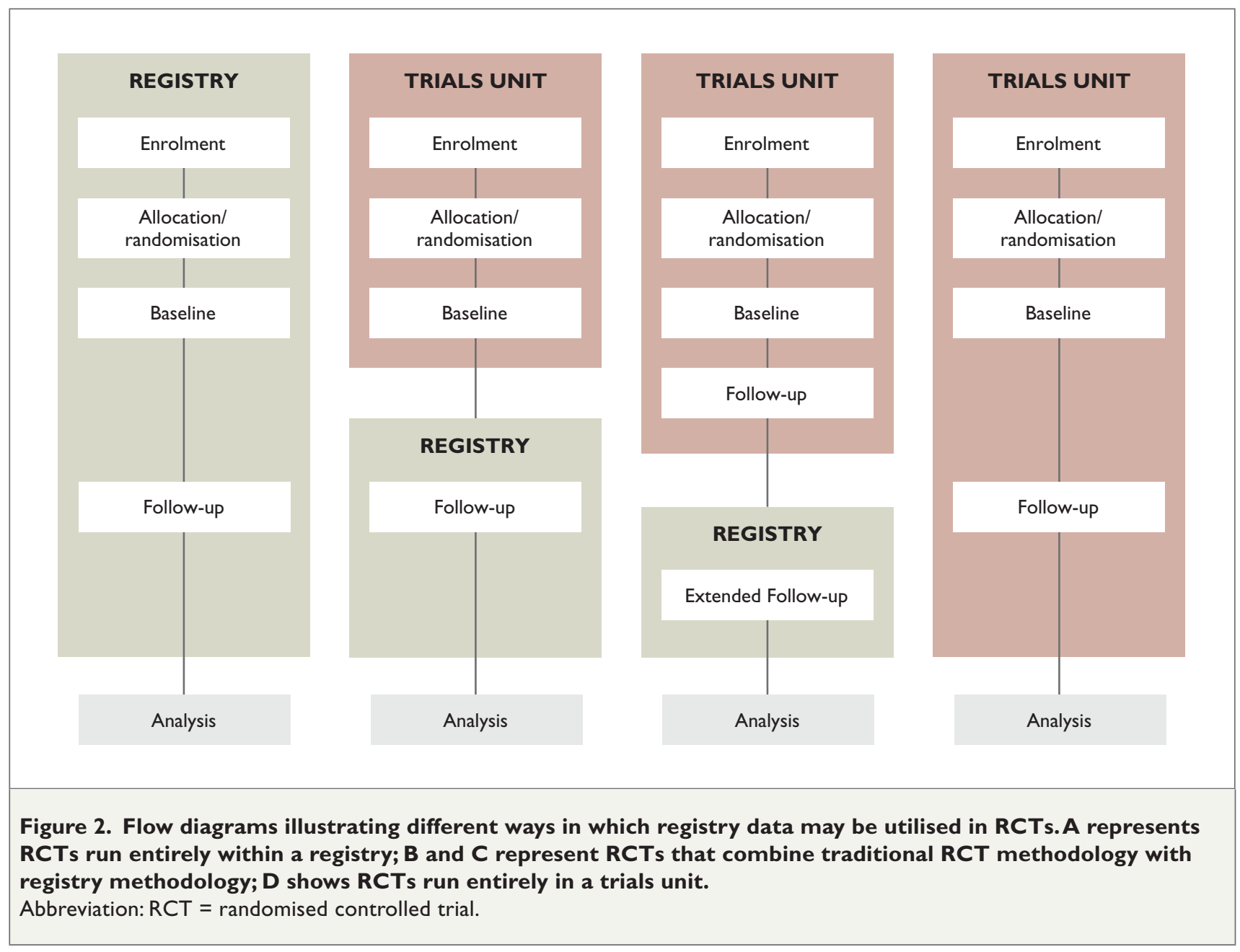

External factors such as adherence with the treatment are also less aggressively controlled and more like what would be expected in the real world. The results from pragmatic registry RCTs are therefore more likely to be observed as the interventions are rolled out into routine practice.

Some registries have permissions to be used to screen for patients who meet the eligibility criteria and so can be used to identify consecutive patients, thus reducing selection bias. Large registry cohorts offer the opportunity to perform nested randomized trials for multiple interventions simultaneously. This is known as a cohort multiple RCT [13]. Most commonly, however, registries are used to provide long-term follow-up and where there is national coverage they bring the major advantage of exceedingly low rates of loss to follow-up [14].

Registry RCTs have their challenges too, however. Broadly speaking, these can be summarised as related to data quality, information governance permissions and methodological challenges.

The primary purpose of most registries is not research, and so the same rigour is not applied to data capture and
RCTs. While data dictionaries may exist, the validity of the data still relies on the reliability of clinical staff routinely entering the data. A great deal of attention therefore needs to be paid to the evidence of completeness and validity of any registry data before deciding on a registry RCT approach [15]. Most obviously, this will influence the choice of end-point and the ability to adjust for baseline confounders. It is also worth reflecting on the registry's ability to routinely monitor adherence to treatment - or components of treatment for a complex intervention - which becomes very pertinent when interpreting the results of the RCT, particularly if the result is null.

For the same reasons, the existing information governance approvals - providing compliance with data protection law and the Declaration of Helsinki - will almost certainly not permit interventional studies. In this regard the landscape differs from jurisdiction to jurisdiction and general advice is hard to give. As well as the usual research ethics approvals, it will be necessary to ensure that all data protection laws are complied with. In some countries, laws are more accommodating than in others, which explains why more than half of all registry RCTs identified in a recent systematic 
review had been conducted in Denmark or Sweden [14] Data protection compliance becomes particularly challenging when data from more than one source need to be linked.

From a methodological perspective there are also several challenges. As discussed above, choice of end-point will be affected by the available data and their quality. Adjudication of end-points will not be the norm, though this may be less important for outcomes arising from routine data [ 16]. For both of these reasons there is likely to be lower precision around the effect estimate in a registry RCT, which will have implications for sample size calculations. While event rates may be greater in real-world data [5], and this may give the impression of greater statistical power, it is important to weigh this against the high chance that events not likely to be affected by the intervention will attenuate any effect.

There are number of ways in which registry data may be incorporated into an RCT study design. It may be possible to carry out enrolment, allocation, randomisation and follow-up within the registry framework. Alternatively, registries may provide follow-up data in traditional RCTs (see Figure 2) [17].

\section{REGISTRY RCTS THAT HAVE BEEN COMPLETED AND SOME THAT ARE UNDER WAY}

In a 2017 systematic review, only 7 I registry RCTs were identified across all specialities [14]. For readers looking for more case studies to learn from, the full list of published registry RCTs is available in the supplementary material of the paper, but a few are worth highlighting.

The Swedish Web System for Enhancement and Development of Evidence-based Care in Heart Disease Evaluated According to Recommended Therapies (SWEDEHEART) registry [ 8 ] provides a powerful illustration of what can be achieved with a registry platform capable of supporting RCTs. SWEDEHEART collects data via a web-based data entry system on consecutive patients presenting with acute coronary syndrome or undergoing coronary angiography/ angioplasty or heart surgery across all 74 hospitals providing this service in Sweden [18]. Each year a validation exercise is undertaken on a random sample of 30-40 cases in 20 of these hospitals. Using this platform, SWEDEHEART has been able to conduct large RCTs looking at highly clinically relevant interventions such as manual thrombus aspiration in patients with acute myocardial infarction undergoing percutaneous intervention (TASTE, 7,244 patients) [19] and bivalirudin or heparin during percuta- neous coronary intervention (VALIDATE-SWEDEHEART, 6,006 patients) [20]. By relying on routine registry data for baseline and follow-up data, SWEDEHEART has an impressive track record of keeping costs low - TASTE reportedly cost only $\$ 350,000$ in total, which equates to $\$ 50$ per recruited patient [2।].

In nephrology, registries have been used to provide longterm follow-up data beyond the end of the original traditional RCT. A recent, but still early, example was the use of linkage to a number of routine databases to provide the outcomes for one of the randomisation arms in the Campath, Calcineurin inhibitor reduction, and Chronic allograft nephropathy (3C) study, which randomised kidney transplant recipients to tacrolimus or sirolimus maintenance therapy [22]. Linking to a number of databases in the UK - Hospital Episode Statistics, Office for National Statistics, UK Transplant and UK Renal Registry - the researchers were able to obtain follow-up data on graft function (serum creatinine), graft failure, patient survival, cancer and cause-specific mortality.

Routine data can also be used to evaluate more complex interventions delivered at a ward, hospital or renal unit level, with randomisation occurring at the level of the 'cluster'. Two such trials addressing kidney health outcomes are worth considering. The first is the Primary-Secondary Care Partnership to Improve Outcomes in Chronic Kidney Disease (PSP-CKD) study, which rolled out a nurse-led CKD management programme aimed at improving outcomes in patients with CKD in primary care [23]. Forty-six primary care practices were provided with the IMPAKT CKD software to help manage CKD before being randomly allocated to receive or not receive an experienced CKD nurse practitioner to support an allocated CKD clinical lead. Outcomes were extracted from the routine primary care data by the IMPAKT software and included change in mean eGFR (at the practice level) over 42 months as well as blood pressure control and recognition of CKD. Although processes of care associated with improved longterm outcomes improved, no significant improvement was observed in the mean eGFR at the practice level [23].

When it might be harder to get sites to agree to being randomised to the control arm, an alternative cluster design is a stepped-wedge cluster RCT. With this approach, all sites are exposed to the intervention, but the order in which they adopt the intervention is determined at random. This was the design of the Tackling Acute Kidney Injury (Tackling AKI) study in which a 3-component complex intervention - AKI e-alerts, a care bundle and an education package - was rolled out across five hospitals in England with the aim of reducing mortality [24]. Despite a promising 
$30 \%$ reduction in hospital-level mortality being observed in a single-centre pilot study, and 24,059 AKI episodes with a 30 -day mortality of $24.5 \%$, the intervention did not appear to have any effect on 30-day mortality in the trial. As with PSP-CKD, however, significant improvements were observed in processes of care and there was evidence of reduced length of hospital stay [24].

Of course, RCTs, even registry RCTs, have a long gestation period and it is worth noting that there are several kidneyfocused registry RCTs which are funded and under way, and expected to report the results in the next few years. For example:

- The Natural vitamin D (cholecalciferol) versus standard care in patients receiving dialysis - The SIMPLIFIED randomised registry trial, which is collecting baseline data traditionally, but with all follow-up through data linkage including the highly innovative linkage with weekly laboratory data feeds from the UK Renal Registry [25]. The study is funded by the National Institute for Health Research, has a recruitment target of 4,200 patients from 36 UK hospitals with 5 years of planned follow-up and has all-cause mortality as the primary outcome.

- The High-volume HDF versus High-flux HD Registry Trial (H4RT), which is collecting baseline data traditionally with research nurses and case report forms, but then manages all follow-up through linkage to routine data [26]. It is funded by the National Institute for Health Research, has a recruitment target of I,550 from 30 UK hospitals with up to 4.5 years of follow-up and has a composite primary end-point of non-cancer death or admission to hospital with a cardiovascular- or infectionrelated event.

- The Better Evidence for Selecting Transplant Fluids (BEST-Fluids) trial, which is evaluating the effect, in deceased donor kidney transplant recipients, of intravenous therapy with Plasmalyte $\AA$ versus $0.9 \%$ saline on delayed graft function following deceased donor kidney transplantation. Participants will be enrolled, randomised and followed up using ANZDATA, the Australia \& New Zealand Dialysis \& Transplant Registry [27]. It is funded by the National Health and Medical Research Council of Australia with a recruitment target of 800 .

\section{CONCLUSIONS}

There are real opportunities to embed RCTs in routinely collected healthcare data and these should be possible in nephrology in particular given the established infrastructure protection laws in some countries make this easier than in others, but if these can be worked through it is possible to create platforms with cohorts from which patients can be screened and assessed for eligibility before being offered participation in these pragmatic trials. Although the relatively low costs look appealing, they rely on investment in and support of the infrastructure - the renal registries.

With all those caveats, registries provide a very exciting opportunity to increase the number of RCTs being undertaken in nephrology and generate better quality evidence for us to improve the quality of care and outcomes for people with kidney disease.

\section{REFERENCES}

I. Guyatt GH, Sackett DL, Sinclair JC, Hayward R, Cook DJ, Cook RJ. Users' guides to the medical literature. IX. A method for grading health care recommendations. Evidence-Based Medicine Working Group. JAMA. 1995; 274:1800-1804.

2. Wilson LM, Rebholz CM, Jirru E, Liu MC, Zhang A, Gayleard J, et al. Benefits and harms of osteoporosis medications in patients with chronic kidney disease: a systematic review and meta-analysis. Ann Intern Med. 2017; 166:649-658.

3. Mathew RO, Bangalore S, Lavelle MP, Pellikka PA, Sidhu MS, Boden WE, et al. Diagnosis and management of atherosclerotic cardiovascular disease in chronic kidney disease: a review. Kidney Int. 2017; 91:797-807.

4. Strippoli GFM, Craig JC, Schena FP. The number, quality, and coverage of randomized controlled trials in nephrology. J Am Soc Nephrol. 2004; I5:4II-419.

5. Smyth B, Haber A, Trongtrakul K, Hawley C, Perkovic V, Woodward M, et al. Representativeness of randomized clinical trial cohorts in end-stage kidney disease: a meta-analysis. JAMA Intern Med. 2019; 179(10):1316-1324.

6. Schwartz D, Lellouch J. Explanatory and pragmatic attitudes in therapeutical trials. J Clin Epidemiol. 2009; 62:499-505.

7. Loudon K, Treweek S, Sullivan F, Donnan P, Thorpe KE, Zwarenstein $M$. The PRECIS-2 tool: designing trials that are fit for purpose. BMJ. 20I5; 350:h2 I47.

8. Speich B, von Niederhausern B, Schur N, Hemkens LG, Furst T, Bhatnagar $N$, et al. Systematic review on costs and resource use of randomized clinical trials shows a lack of transparent and comprehensive data. J Clin Epidemiol. 2018; 96: I- II.

9. Verkooijen $\mathrm{H}$, Roes $\mathrm{K}$. Cohort multiple randomized controlled trial: a solution for the evaluation of multiple interventions. Ned Tijdschr Geneeskd. 20 13; 157:A5762-A5762.

10. Zwarenstein M, Treweek S, Gagnier J), Altman DG, Tunis S, Haynes B, et al. Improving the reporting of pragmatic trials: an extension of the CONSORT statement. BMJ. 2008; 337:a2390.

I I. Dal-Ré R, Janiaud P, loannidis JP. Real-world evidence: How pragmatic are randomized controlled trials labeled as pragmatic? BMC Med. 2018; 16:49.

12. Irving E, van den Bor R, Welsing P, Walsh V, Alfonso-Cristancho R, Harvey C, et al. Series: Pragmatic trials and real world evidence: Paper 7. Safety, quality and monitoring. I Clin Epidemiol. 2017; 91:6-12.

13. Relton C, Torgerson D, O'Cathain A, Nicholl J. Rethinking pragmatic randomised controlled trials: introducing the "cohort multiple 
randomised controlled trial" design. BMJ. 20 I 0; 340:c 1066.

14. Mathes T, Buehn S, Prengel P, Pieper D. Registry-based randomized controlled trials merged the strength of randomized controlled trails and observational studies and give rise to more pragmatic trials. J Clin Epidemiol. 2018; 93: I 20- 127.

15. Lauer MS, D'Agostino RB, Sr. The randomized registry trial - the next disruptive technology in clinical research? N Engl J Med. 20I3; 369:|579-|58|

16. Ndounga Diakou LA, Trinquart L, Hrobjartsson A, Barnes C, Yavchitz A, Ravaud P, et al. Comparison of central adjudication of outcomes and onsite outcome assessment on treatment effect estimates. Cochrane Database Syst Rev. 2016; 3:MR000043.

17. Nyberg K, Hedman P. Swedish guidelines for registry-based randomized clinical trials. Ups J Med Sci. 2019; 124:33-36.

18. Jernberg T, Attebring MF, Hambraeus K, Ivert T, James S, Jeppsson A, et al. The Swedish Web-system for enhancement and development of evidence-based care in heart disease evaluated according to recommended therapies (SWEDEHEART). Heart. 2010; 96:1617-1621.

19. Frobert O, Lagerqvist B, Olivecrona GK, Omerovic E, Gudnason T, Maeng $\mathrm{M}$, et al. Thrombus aspiration during ST-segment elevation myocardial infarction. N Engl J Med. 2013; 369:1587-I597.

20. Erlinge D, Omerovic E, Frobert O, Linder R, Danielewicz M, Hamid M, et al. Bivalirudin versus heparin monotherapy in myocardial infarction. N Engl J Med. 2017; 377: | | 32-1 | 42.

2I. Wachtell K, Lagerqvist B, Olivecrona GK, James SK, Fröbert O. Novel trial designs: lessons learned from thrombus aspiration during ST-segment elevation myocardial infarction in Scandinavia (TASTE) trial. Curr Cardiol Rep. 2016; 18:1।.

22. The 3C Study Collaborative Group. Campath, calcineurin inhibitor reduction, and chronic allograft nephropathy (the 3 C Study) - results of a randomized controlled clinical trial. Am J Transplant. 20 8; | 8:1424- 4434.

23. Major RW, Brown C, Shepherd D, Rogers S, Pickering W, Warwick GL, et al. The primary-secondary care partnership to improve outcomes in chronic kidney disease (PSP-CKD) study: a cluster randomized trial in primary care. J Am Soc Nephrol. 2019; 30:1261-1270.

24. Selby NM, Casula A, Lamming L, Stoves J, Samarasinghe Y, Lewington AJ, et al. An organizational-level program of intervention for AKI: a pragmatic stepped wedge cluster randomized trial. J Am Soc Nephrol. 2019; 30:505-5 I 5.

25. Natural vitamin D (cholecalciferol) versus standard care in patients receiving dialysis - The SIMPLIFIED randomised registry trial. http:// www.isrctn.com/ISRCTNI50876I6. Accessed 23 October 2019.

26. The High-volume Haemodiafiltration vs High-flux Haemodialysis Registry Trial. https://www.journalslibrary.nihr.ac.uk/programmes/ hta/I 58052/\#/. Accessed 02 January 2018.

27. Better Evidence for Selecting Transplant Fluids (BEST-Fluids). www.aktn.org.au/best-fluids. Accessed 18 July 2019. 